\title{
Nonlinear dynamics of atomic force microscopy with intermittent contact
}

\author{
Yin Zhang, Ya-pu Zhao * \\ State Key Laboratory of Nonlinear Mechanics (LNM), Institute of Mechanics, Chinese Academy of Sciences, \\ Beijing 100080, People's Republic of China
}

Accepted 31 March 2006

\begin{abstract}
When the atomic force microscopy (AFM) in tapping mode is in intermittent contact with a soft substrate, the contact time can be a significant portion of a cycle, resulting in invalidity of the impact oscillator model, where the contact time is assumed to be infinitely small. Furthermore, we demonstrate that the AFM intermittent contact with soft substrate can induce the motion of higher modes in the AFM dynamic response. Traditional ways of modeling AFM (one degree of freedom (DOF) system or single mode analysis) are shown to have serious mistakes when applied to this kind of problem. A more reasonable displacement criterion on contact is proposed, where the contact time is a function of the mechanical properties of AFM and substrate, driving frequencies/amplitude, initial conditions, etc. Multi-modal analysis is presented and mode coupling is also shown.
\end{abstract}

(c) 2006 Published by Elsevier Ltd.

\section{Introduction}

Modeling AFM structure as one DOF system or single mode analysis offers a simple way of analyzing AFM dynamic behavior and in certain scenarios this simple modeling catches the essential AFM dynamic characteristics [1-6]. Burham et al. [7] are the first to apply impact oscillator model to the problem of AFM intermittent contact with the (hard) substrate/sample in tapping mode [2]. However, the impact oscillator model assumes the infinitely small contact time [8], which physically means the oscillator bounces back instantaneously. Therefore, impact oscillator model is only suitable for the case of AFM contact with the hard substrate/sample. When AFM is in intermittent contact with the relatively soft substrate and even the driving frequency is lower than or around the system fundamental frequency, one DOF or single mode analysis should not be used without extreme caution. The discontinuity of AFM system stiffness caused by intermittent contact is a major source of nonlinearity. The intermittent contact together with the driving frequency and amplitude can induce the motion of higher modes in AFM dynamic response. This paper demonstrates this through a multi-modal analysis approach.

\footnotetext{
* Corresponding author. Tel.: +86106265 8008; fax: +86 10626561284 .

E-mail address: yzhao@lnm.imech.ac.cn (Y.-p. Zhao).
} 


\section{Model development}

In Fig. 1(a), the AFM cantilever beam neutral axis and tip are initially separated from the sample with the distance of $g_{0}$ and $g_{1}$, respectively. AFM is driven with a forced motion $y(t)(t$ is time). $w(x, t)$ in Fig. 1(b) is the beam deflection from the fixed end. When the AFM tip hits the sample, AFM tip is modeled to be in contact with a spring with the stiffness of $K_{1}$ and a damper with the viscous damping of $C_{1}$. The cantilever beam displacement $v(x, t)$ and tip displacement $v_{T}(x, t)$ in the coordinate system shown in Fig. 1(b) are as follows:

$$
v(x, t)=w(x, t)+y(t)+g_{0}, \quad v_{T}(x, t)=v_{T}(t)=v(L, t)-\left(g_{0}-g_{1}\right)=w(L, t)+y(t)+g_{1} .
$$

$L$ is the beam length. By applying Hamilton's principle, the equation of motion is derived as follows:

$$
\begin{cases}m \frac{\partial^{2} v}{\partial t^{2}}+C \frac{\partial v}{\partial t}+E I \frac{\partial^{4} v}{\partial x^{4}}=0 & v_{T}>0 \\ m \frac{\partial^{2} v}{\partial t^{2}}+C \frac{\partial v}{\partial t}+E I \frac{\partial^{4} v}{\partial x^{4}}+K_{1} \delta(x-L)\left[w(x, t)+y(t)+g_{1}\right]+C_{1} \delta(x-L) \frac{\partial v}{\partial t}=0, & v_{T} \leqslant 0\end{cases}
$$

$m$ is the mass per unit length of the beam. $C$ is the viscous damping of the beam. $E$ is Young's modulus and $I=\frac{b h^{3}}{12}(b$ : beam width, $h$ : beam height) for the beam with rectangular cross-section. Here $\delta$ is Dirac delta function. $v_{T}$ defines the switching condition. $y(t)$ is assumed as a sinusoidal motion of $y(t)=f \sin (\omega t)$. $f$ and $\omega$ are the driving amplitude and frequency, respectively. In order to nondimensionalize Eq. (2), the following dimensionless quantities are introduced:

$$
\xi=\frac{x}{L}, \quad \tau=\sqrt{\frac{E I}{m L^{4}}} t, \quad W=\frac{w}{g_{0}}, \quad V=\frac{v}{g_{0}}, \quad V_{T}=\frac{v_{T}}{g_{0}}, \quad \Omega=\sqrt{\frac{m L^{4}}{E I}} \omega .
$$

The dimensionless governing equations of $W$ are now

$$
\begin{cases}\frac{\partial^{2} W}{\partial \tau^{2}}+\alpha_{2} \frac{\partial W}{\partial \tau}+\frac{\partial^{4} W}{\partial \xi^{4}}=\alpha_{1} \Omega^{2} \sin (\Omega \tau)-\alpha_{1} \alpha_{2} \Omega \cos (\Omega \tau) & V_{T}>0 \\ \frac{\partial^{2} W}{\partial \tau^{2}}+\alpha_{2} \frac{\partial W}{\partial \tau}+\frac{\partial^{4} W}{\partial \xi^{4}}+\alpha_{3} \delta(\xi-1) W+\alpha_{5} \delta(\xi-1) \frac{\partial W}{\partial \tau}=\alpha_{1} \Omega^{2} \sin (\Omega \tau)-\alpha_{1} \alpha_{2} \Omega \cos (\Omega \tau) & >\alpha_{T} \leqslant 0 \\ -\alpha_{1} \alpha_{3} \delta(\xi-1) \sin (\Omega \tau)-\alpha_{3} \alpha_{4} \delta(\xi-1)-\alpha_{1} \alpha_{5} \delta(\xi-1) \Omega \cos (\Omega \tau), & V(\xi-1)\end{cases}
$$

Here $\alpha_{i} \mathrm{~s}(i=1-5)$ are defined as:

$$
\alpha_{1}=\frac{f}{g_{0}}, \quad \alpha_{2}=C \sqrt{\frac{L^{4}}{m}}, \quad \alpha_{3}=\frac{K_{1} L^{3}}{E I}, \quad \alpha_{4}=\frac{g_{1}}{g_{0}}, \quad \alpha_{5}=\frac{C_{1}}{L} \sqrt{\frac{L^{4}}{m}} .
$$

$W(\xi, \tau)$ is assumed to have the following form:

$$
W(\xi, \tau)=\sum_{j=1}^{N} a_{j}(\tau) \phi_{i}(\xi)
$$

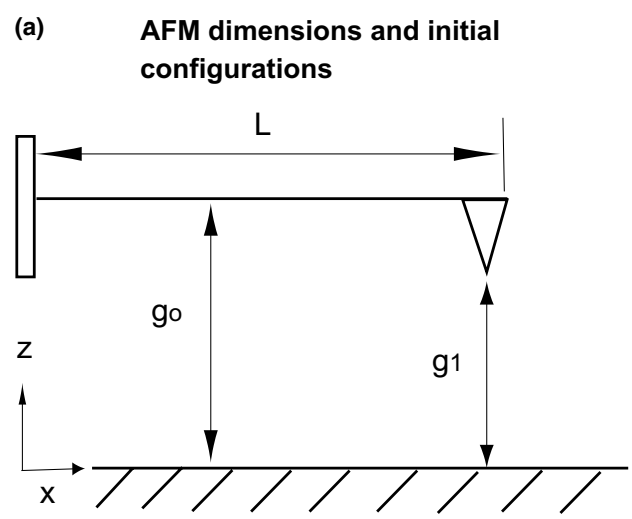

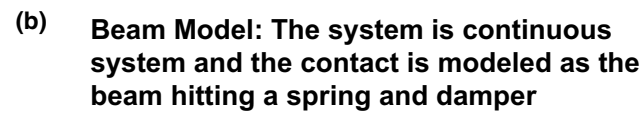

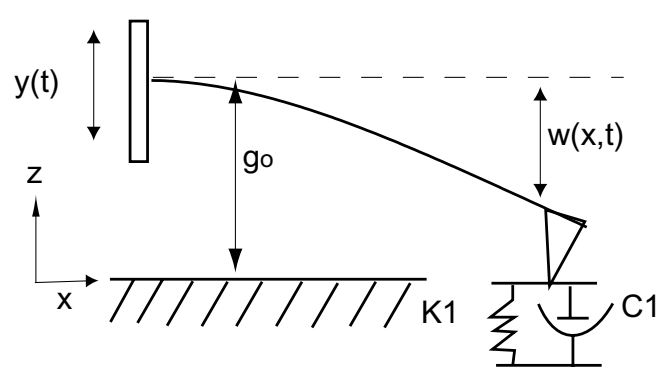

Fig. 1. (a) AFM dimensions and initial configurations and (b) the cantilever beam modeling of AFM. When AFM contacts the substrate, it is modeled as AFM hitting a spring and a damper. 
$N$ is the mode number, $\phi_{j}(\xi)$ is the mode shape of cantilever beam. Galerkin method which substitutes Eq. (6) into Eq. (4), times $\phi_{i}(\xi)$ and integrates from 0 to 1 is then applied [9]. Fourth order Runge-Kutta integration is used for the time integration of Eq. (4).

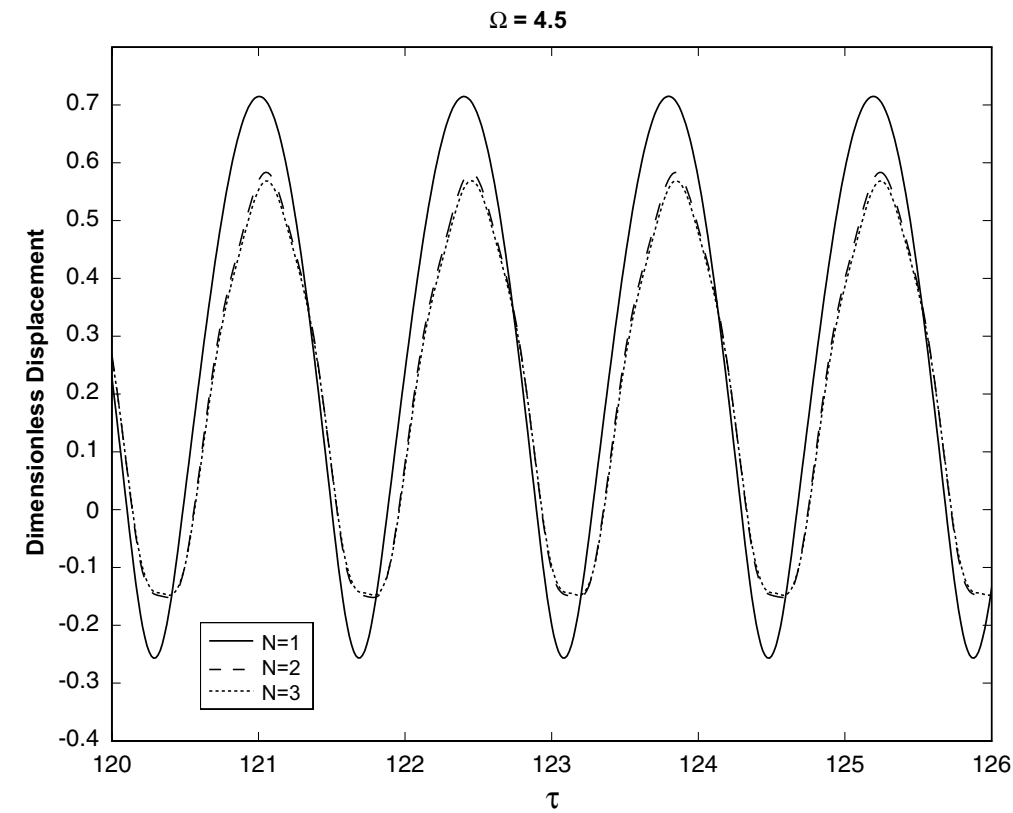

Fig. 2. The convergence study of AFM motion computed with different mode numbers at $\Omega=4.5$.

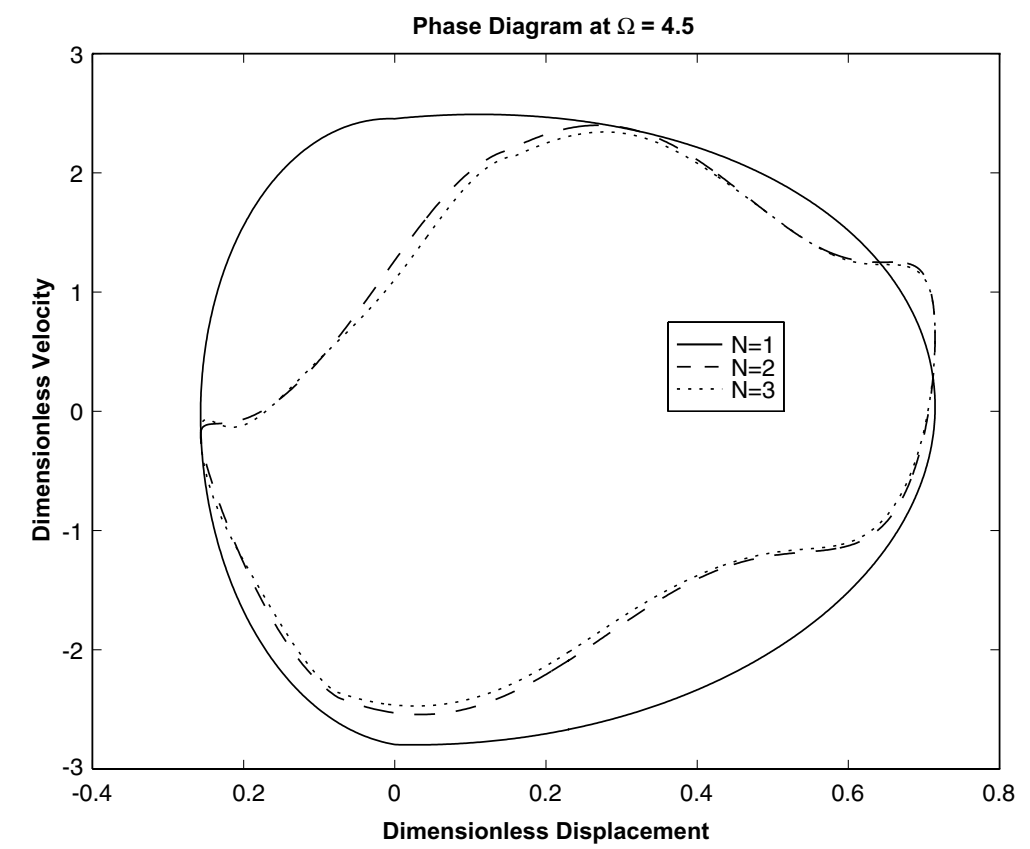

Fig. 3. Phase diagram comparison computed by different mode numbers at $\Omega=4.5$. 


\section{Results discussion and remarks}

In Figs. 2 and 3, the following parameters are taken:

$$
\alpha_{1}=0.1, \quad \alpha_{2}=0.1, \quad \alpha_{3}=20, \quad \alpha_{4}=0, \quad \alpha_{5}=0.2 .
$$

Fig. 2 shows the time series of AFM motion. That $\tau$ starts from 120 is to guarantee that AFM system is in the steady state. The driving frequency is around the fundamental frequency and far away from the second one. Clearly at $\alpha_{3}=20$ of soft substrate, the computation results of 1 mode are significantly different with those of 2 or 3 modes. So are the phase diagrams in Fig. 3. The computation results of 2 modes agree well with those of 3 modes. This physically means that the contribution of the second mode to the AFM motion is significant and the third one is not.

The motion of higher mode(s) in the AFM dynamic response even when the driving frequency is around the fundamental one is shown. Subharmonic motion which is one of major dynamic characteristics in intermittent contact dynamics is not shown in this paper. However, the information of subharmonic motion is included in Eq. (2) and further exploration on subharmonic motion is now carried out in our future work.

\section{Acknowledgements}

Zhang is thankful to the financial supports from both the National Natural Science Foundation of China (NSFC, Grant No. 10502050) and the Scientific Research Foundation for the Returned Overseas Chinese Scholars, State Education Ministry. Zhao is supported by the Distinguished Young Scholar Fund of the National Natural Science Foundation of China (NSFC, Grant No. 10225209), key project from Chinese Academy of Sciences (Grant No. KJCX-SWL2) and NSFC project (Grant No. 90305020).

\section{References}

[1] Lee SI, Howell SW, Raman A, Reifenberger R. Nonlinear dynamics of microcantilevers in tapping mode atomic force microscopy: A comparison between theory and experiment. Phys Rev B 2002;66:115409.

[2] van de Water W, Molenaar J. Dynamics of vibrating atomic force microscopy. Nanotechnology 2000;11:192-9.

[3] Aimé JP, Boisgard R, Nony L, Couturier G. Nonlinear dynamic behavior of an oscillating tip-microlever system and contrast at the atomic scale. Phys Rev Lett 1999;82:3388-91.

[4] Nony L, Boisgard R, Aimé JP. Nonlinear dynamical properties of an oscillating tip-cantilever system in tapping mode. J Chem Phys 1999;111:1615-27.

[5] Berg J, Briggs GAD. Nonlinear dynamics of intermittent-contact mode atomic force microscopy. Phys Rev B 1997;55:14899-908.

[6] Garcia R, Paulo AS. Dynamics of a vibrating tip near or in intermittent contact with a surface. Phys Rev B 2000;61:R13381-4.

[7] Burham NA, Kulik AJ, Gremaud G, Briggs GAD. Nanosubharmonics: The dynamics of small nonlinear contacts. Phys Rev Lett 1995;74:5092-5.

[8] Holmes PJ. The dynamics of repeated impacts with a sinusoidally vibrating table. J Sound Vib 1982;84:173-89.

[9] Murphy KD, Zhang Y. Vibration and stability of a cracked translating beam. J Sound Vib 2000;237:319-35. 